\title{
Predicting the survival probability of gastric cancer patients developing metastatic epidural spinal cord compression (MESCC)
}

\author{
Dirk Rades • Stefan Huttenlocher • Tobias Bartscht • \\ Steven E. Schild
}

Received: 26 June 2014/ Accepted: 29 December 2014/Published online: 15 January 2015

(C) The International Gastric Cancer Association and The Japanese Gastric Cancer Association 2015

\begin{abstract}
To create a tool for estimating the survival of gastric cancer patients developing MESCC, clinical factors were evaluated in 29 patients. Factors were age, gender, performance status, affected vertebrae, ambulatory status, further bone metastases, visceral metastases, time from gastric cancer diagnosis until MESCC and rapidity of developing weakness of legs. On multivariate analyses, visceral metastases (risk ratio: 6.80; $p=0.003$ ) and rapidity of weakening of legs (risk ratio: $2.73 ; p=0.023$ ) had a significant effect on survival and were included in the tool. Scoring points for each of the two factors were either 0 or 1 , depending on the 6-month survival rates. According to the sum of the points, three groups were built: 0 points $(n=12), 1$ point $(n=10)$ and 2 points $(n=7)$. Sixmonth survival rates were 0,20 and $100 \%(p<0.001)$. This tool for patients with MESCC from gastric cancer estimates survival probabilities, which is important for tailoring treatment to patients' needs.
\end{abstract}

Keywords Gastric cancer - Metastatic epidural spinal cord compression - Irradiation - Survival - Predictive tool

D. Rades $(\bowtie) \cdot S$. Huttenlocher

Department of Radiation Oncology, University of Lübeck, Ratzeburger Allee 160, 23538 Lübeck, Germany

e-mail: rades.dirk@gmx.net

T. Bartscht

Department of Hematology and Medical Oncology, University of Lübeck, Lübeck, Germany

S. E. Schild

Department of Radiation Oncology, Mayo Clinic,

Scottsdale, AZ, USA

\section{Introduction}

Gastric cancer patients account for 1-2\% of patients developing metastatic epidural spinal cord compression (MESCC) [1, 2]. Since the treatment of locoregional disease is constantly improving, the number of patients with MESCC from gastric cancer will increase. MESCC is a serious situation and considered an oncologic emergency. Irradiation alone is the most common treatment. Upfront surgery was shown to improve the outcomes of selected patients with a good performance status and favorable survival prognosis [3]. Therefore, it is important to judge a patient's remaining lifetime as precisely as possible. If irradiation alone is administered, different schedules are available. These include single-fraction (1 day), shorter course (1 week) and longer course programs (2-4 weeks) [4]. The most common schedule worldwide is $10 \times 3 \mathrm{~Gy}$ in 2 weeks. For patients with a very limited lifespan, the overall time of irradiation should be as short as possible and generally not exceed 1 week. Patients with a much more favorable prognosis were suggested to benefit from longer course programs with total doses $>30 \mathrm{~Gy}$ in terms of improved outcomes [5]. Selected patients with an extraordinarily good prognosis may be considered for stereotactic body radiotherapy or radiosurgery [6].

These various treatment options and their proper selection exemplify the importance of being able to forecast the survival time of patients developing MESCC. Although several survival scores have been reported, none was particularly designed for gastric cancer patients [7]. This study was performed to close this gap. Since every primary tumor leading to MESCC is different from others regarding its biology and clinical course, optimal personalization of MESCC treatment can be best realized if the expected survival duration is adequately estimated and appreciated. 


\section{Materials and methods}

Twenty-nine patients irradiated for MESCC from gastric cancer were included in this retrospective study. Seven patients $(24 \%)$ received short-course irradiation with $1 \times 8$ in 1 day or $5 \times 4$ Gy in 1 week and 22 patients $(76 \%)$ longer course irradiation with $10 \times 3 \mathrm{~Gy}$ in 2 weeks, $15 \times 2.5 \mathrm{~Gy}$ in 3 weeks or $20 \times 2 \mathrm{~Gy}$ in 4 weeks. To reduce the risk of a selection bias due to different treatments, patients receiving spinal surgery were not included. All patients experienced weakness of the legs. They did not have prior local treatment to the involved spinal cord and were seen by a surgeon before irradiation. MESCC was diagnosed by computed tomography or magnetic resonance imaging. Dexamethasone was started immediately and tapered down after radiotherapy. Radiation was administered with 6-10-MV photon beams from a linear accelerator, encompassing one normal vertebra cranial and caudal from those affected by metastasis.

Nine pretreatment clinical factors were evaluated for effects on survival. These factors included age ( $<65$ vs. $\geq 65$ years, median: 65.5 years), gender, performance status according to the Eastern Cooperative Oncology Group score (ECOG 1-2 vs. 3-4), number of involved vertebrae (1-2 vs. $\geq 3$ vertebrae), ambulatory status before irradiation (ambulatory vs. not ambulatory), further bone metastases at the time of irradiation (no vs. yes), visceral metastases at the time of irradiation (no vs. yes), time from diagnosis of gastric cancer until MESCC ( $\leq 15$ vs. $>15$ months, median: 15 months) and rapidity of developing weakness of the legs before irradiation (faster: $\leq 14$ days vs. slower: $>14$ days) (Table 1). Univariate analysis was performed with the Kaplan-Meier method and the log-rank test. According to Bonferroni correction for multiple tests, findings were significant for $p<0.005$ representing an alpha level of $<0.05$. Factors that were significant on univariate analysis were subsequently included in multivariate analyses (Cox proportional hazards model).

\section{Results}

Four clinical factors were found to have a significant association with survival on univariate analysis (Table 1),
Table 1 Survival rates at 3 and 6 months

According to Bonferroni correction, $p$ values $<0.005$ were considered significant

\begin{tabular}{|c|c|c|c|}
\hline & At 3 months $(\%)$ & At 6 months $(\%)$ & $p$ \\
\hline \multicolumn{4}{|l|}{ Age } \\
\hline$<65$ years $(n=14)$ & 64 & 36 & \\
\hline$\geq 65$ years $(n=15)$ & 47 & 27 & 0.60 \\
\hline \multicolumn{4}{|l|}{ Gender } \\
\hline Female $(n=11)$ & 45 & 36 & \\
\hline Male $(n=18)$ & 61 & 28 & 0.94 \\
\hline \multicolumn{4}{|l|}{ ECOG performance score } \\
\hline $1-2(n=13)$ & 85 & 62 & \\
\hline $3-4(n=16)$ & 31 & 6 & $<0.001$ \\
\hline \multicolumn{4}{|l|}{ Number of involved vertebrae } \\
\hline $1-2$ vertebrae $(n=11)$ & 64 & 45 & \\
\hline$\geq 3$ vertebrae $(n=18)$ & 50 & 22 & 0.18 \\
\hline \multicolumn{4}{|c|}{ Ambulatory status before irradiation } \\
\hline Ambulatory $(n=16)$ & 81 & 56 & \\
\hline Not ambulatory $(n=13)$ & 23 & 0 & $<0.001$ \\
\hline \multicolumn{4}{|l|}{ Further bone metastases } \\
\hline No $(n=12)$ & 67 & 50 & \\
\hline Yes $(n=17)$ & 47 & 18 & 0.064 \\
\hline \multicolumn{4}{|l|}{ Visceral metastases } \\
\hline No $(n=9)$ & 100 & 78 & \\
\hline Yes $(n=20)$ & 35 & 10 & $<0.001$ \\
\hline \multicolumn{4}{|c|}{ Time from diagnosis of gastric cancer until MESCC } \\
\hline$\leq 15$ months $(n=15)$ & 53 & 27 & \\
\hline$>15$ months $(n=14)$ & 57 & 36 & 0.71 \\
\hline \multicolumn{4}{|c|}{ Rapidity of developing weakness of legs } \\
\hline Faster $(\leq 14$ days $)(n=14)$ & 29 & 0 & \\
\hline Slower $(>14$ days $)(n=15)$ & 80 & 60 & $<0.001$ \\
\hline
\end{tabular}


Table 2 Six-month survival rates and scoring points (survival rates divided by 10)

\begin{tabular}{lll}
\hline & Survival at 6 months (\%) & Scoring points \\
\hline Visceral metastases & & \\
No & 78 & 1 \\
Yes & 10 & 0 \\
Rapidity of developing & weakness of legs & \\
Faster ( $\leq 14$ days $)$ & 0 & 0 \\
Slower (>14 days) & 60 & 1 \\
\hline
\end{tabular}

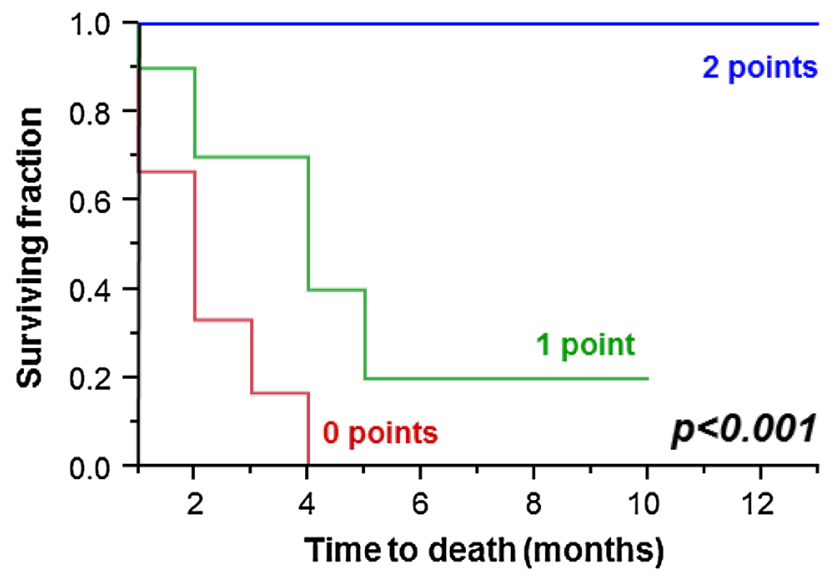

Fig. 1 The Kaplan-Meier curves for survival of the three prognostic groups: 0,1 and 2 points. The $p$ value was calculated with the logrank test

ECOG performance score $(p<0.001)$, pre-irradiation ambulatory status $(p<0.001)$, visceral metastases $(p<0.001)$ and rapidity of developing weakness of the legs $(p<0.001)$. Since the ECOG performance score and pre-irradiation ambulatory status were confounding variables, two multivariate analyses were performed, one including the ECOG performance score and a second one including the preirradiation ambulatory status. On multivariate analyses, visceral metastases [risk ratio (RR): 6.80 ; $95 \%$ confidence interval (CI) 1.83-44.06; $p=0.003]$ and rapidity of developing weakness of the legs (RR: 2.73; $95 \%$ CI 1.13-12.05; $p=0.023$ ) maintained significance. The ECOG performance score (RR: $1.75 ; 95 \%$ CI $0.26-33.33 ; p=0.60)$ and pre-irradiation ambulatory status (RR: 2.33 ; $95 \%$ CI $0.43-14.08 ; p=0.33$ ) were not significant. Visceral metastases and rapidity of developing weakness of the legs were included in the survival score. Scoring points for each of the two factors were either 0 or 1 , depending on the 6-month survival rates (Table 2). Addition of the points for the two factors resulted in the prognostic score for each patient. Prognostic scores were 0 points $(n=12), 1$ point $(n=10)$ or 2 points $(n=7)$. Sixmonth survival rates of these three groups were 0,20 and
$100 \%$, respectively ( $p<0.001$, Fig. 1$)$. Median survival times were 2,4 and $>12$ months (median not reached), respectively.

\section{Discussion}

Metastatic cancer is a specific situation that requires a personalized treatment taking into account patients' individual needs. To optimally tailor the treatment, one has to consider the patient's remaining lifespan. If it is short, late sequelae and long-term local control of MESCC play a minor role, whereas it is important to avoid burdensome and time-consuming procedures. Since functional outcome is similar after short-course irradiation when compared to longer schedules lasting 2-4 weeks, the poor-prognosis patients are sufficiently treated with schedules taking 1 week or less [4].

If the remaining lifetime is longer than a very few months, local control and late morbidity become more important [8]. Selected patients may also benefit from upfront surgery in addition to longer course irradiation. A small randomized study suggested a higher posttreatment ambulation rate ( 84 vs. $57 \%, p=0.001$ ) for patients with a good performance status, favorable survival prognosis, MESCC from a solid tumor and involvement of only one spinal segment [3]. In patients with a survival probability considerably longer than 6 months, a matched-pair study of 382 patients found better results for doses of 37.5-40 Gy compared to $30 \mathrm{~Gy}$ regarding local control of MESCC ( $p=0.012)$ and survival $(p=0.032)$ [5]. To minimize the risk of late morbidity, these patients may be considered for high-precision irradiation with stereotactic body radiotherapy, radiosurgery or intensity-modulated radiotherapy. However, it has been recommended to use these techniques only within clinical trials [6].

Considering available treatments, it becomes obvious that the survival prognosis is an important determinant when designing an individual treatment approach. This also applies to patients with MESCC from gastric cancer. Therefore, we created a predictive tool specifically for this group. Two clinical factors, visceral metastases and rapidity of developing weakness of the legs, had significant associations with survival and were included in the predictive tool. Based on these factors, three survival groups were formed. When using this new tool, one has to bear in mind the retrospective nature of the data included and the relatively small number of patients. Since gastric cancer patients with MESCC are currently quite rare, a predictive tool developed from a larger prospective cohort will not be available soon.

Of patients with 0 points, no one survived for 6 months; median survival was only 2 months. These patients should 
Fig. 2 Algorithm for the treatment of MESCC from gastric cancer

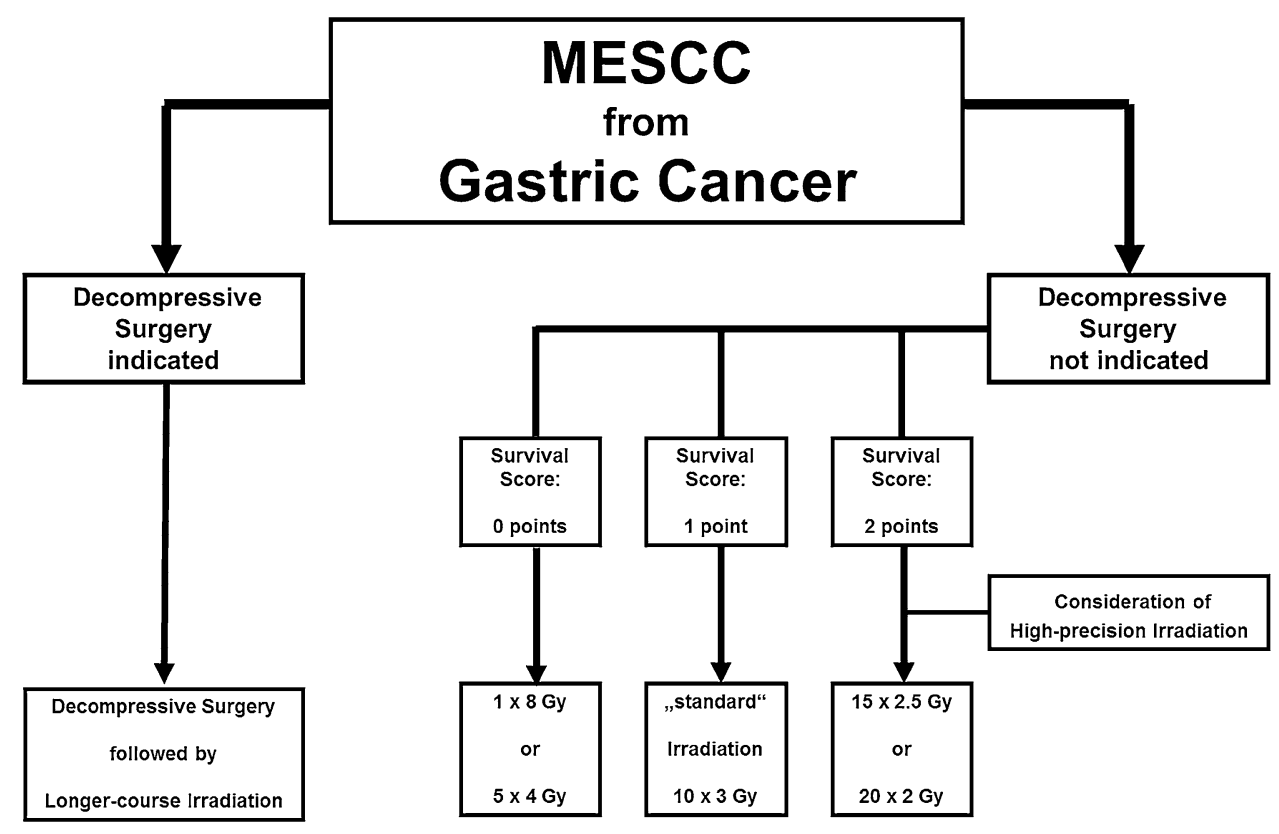

receive short-course irradiation [4]. Of patients achieving 1 point, $20 \%$ survived at least 6 months; median survival was 4 months. These patients may be considered candidates for "standard" irradiation with $10 \times 3$ Gy [4, 8]. Selected patients may receive up-front surgery [3]. All patients achieving 2 points survived longer than 6 months. Median survival time was $>12$ months. These patients should receive longer course irradiation with doses $>30$ Gy [5]. For properly selected patients, up-front surgery should be considered as well as high-precision irradiation (preferably within clinical trials) $[3,6]$. These recommendations are summarized in an algorithm shown in Fig. 2.

In conclusion, this new predictive tool helps to estimate the lifetime of gastric cancer patients developing MESCC. This tool makes it is easier to decide which irradiation schedule is the best and whether upfront surgery and highprecision irradiation should be considered.

\section{References}

1. Prasad D, Schiff D. Malignant spinal cord compression. Lancet Oncol. 2005;6:15-24.
2. Rades D, Abrahm JL. The role of radiotherapy for metastatic epidural spinal cord compression. Nat Rev Clin Oncol. 2010;7: 590-8.

3. Patchell R, Tibbs PA, Regine WF, Payne R, Saris S, Kryscio RJ, et al. Direct decompressive surgical resection in the treatment of spinal cord compression caused by metastatic cancer: a randomised trial. Lancet. 2005;366:643-8.

4. Rades D, Stalpers LJ, Veninga T, Schulte R, Hoskin PJ, Obralic N, et al. Evaluation of five radiation schedules and prognostic factors for metastatic spinal cord compression. J Clin Oncol. 2005;23: 3366-75.

5. Rades D, Panzner A, Rudat V, Karstens JH, Schild SE. Dose escalation of radiotherapy for metastatic spinal cord compression (MSCC) in patients with relatively favorable survival prognosis. Strahlenther Onkol. 2011;187:729-35.

6. Lutz S, Berk L, Chang E, Chow E, Hahn C, Hoskin P, et al. Palliative radiotherapy for bone metastases: an ASTRO evidencebased guideline. Int J Radiat Oncol Biol Phys. 2011;79:965-76.

7. Rades D, Douglas S, Veninga T, Stalpers LJA, Hoskin PJ, Bajrovic A, et al. Validation and simplification of a score predicting survival in patients irradiated for metastatic spinal cord compression. Cancer. 2010;116:3670-3.

8. Rades D, Lange M, Veninga T, Stalpers LJA, Bajrovic A, Adamietz IA, et al. Final results of a prospective study comparing the local control of short-course and long-course radiotherapy for metastatic spinal cord compression. Int J Radiat Oncol Biol Phys. 2011;79:524-30. 